\section{Democratización de la acción pública en la perspectiva de los acuerdos familiares}

Paulo Henrique Martins*

\section{Palabras clave}

Familia, acción pública, democracia, redes sociales, participación

* Sociólogo, profesor Universidade Federal de Pernambuco (UFPE) e investigador Conselho Nacional de Pesquisa (CNPq). Fundador y co-coordinador del Núcleo de Estudios sobre Ciudadanía, Exclusión y Procesos de Cambio (NUCEM), Programa de Posgrado en Sociología, UFPE.

pahem@terra.com.br
Resumen. ¿Tiene la idea de familia algún interés estratégico para pensar en la descentralización y la democratización de la acción pública? Consideramos que sí, siempre y cuando la familia no sea vista como categoría antropológica genérica, definida por la consanguinidad, como propone la antropología tradicional, o referida a la reproducción de la fuerza de trabajo, como lo hace la sociología (Bilac 2003). De hecho, las transformaciones conocidas por el grupo familiar a lo largo de las últimas décadas y los nuevos estudios sobre el tema, han conducido a una importante revisión teórica. Se puede decir que la familia pasa a ser valorada como institución social relevante en la organización de las acciones públicas y en la propia idea de la democratización. De hecho, como intentaremos explicar, es un lugar estratégico para pensar no sólo en la trama de la sexualidad y la reproducción, sino sobre todo - lo que se torna evidente ahora, en el contexto posnacional - en la trama de la normalización del orden social. Si la familia era un tema menor en la organización de las instituciones sociales en general, obviamente se asumía como tema secundario en la organización de las instituciones públicas. El nuevo estatus sociológico adquirido por el asunto viene entre tanto impactando significativamente a los estudiosos de las políticas públicas y a los planificadores y gestores, lo que en Brasil es evidente al estudiar las reformas en la salud pública. 


\section{Introducción}

¿Tiene la idea de familia algún interés estratégico para pensar en la descentralización y la democratización de la acción pública? Consideramos que sí, siempre y cuando la familia no sea vista como categoría antropológica genérica, definida por la consanguinidad, como es propuesta por la antropología tradicional, o referida a la reproducción de la fuerza de trabajo, como es pensada por la sociología (Bilac 2003). De hecho, las transformaciones conocidas por el grupo familiar a lo largo de las últimas décadas y los nuevos estudios sobre el tema, han conducido a una importante revisión teórica.

Claude Lévi-Strauss (2003) se constituye en referencia central en esa revisión, al realzar la relevancia simbólica del parentesco en la organización de la cultura. El avance de los estudios culturales, por su parte, contribuyó decisivamente a deshacer la visión de la cultura como factor secundario en la organización de la sociedad (Williams 1981).

En América Latina, en particular durante las últimas dos décadas, la crisis del paradigma desarrollista facilitó la revalorización de la cultura, llevando a elaborarse una nueva comprensión sobre la cotidianidad y la familia (Jelin 1993). En la actualidad está en curso una nueva profundización de los estudios sobre la familia, a partir del reconocimiento de la relevancia de esta institución, para avanzar en el tema de la participación y la democratización de la acción pública. Tal preocupación se justifica, entre otros motivos, por el hecho de que las políticas dirigidas hacia la familia — nos recuerda Parry Scout — no han merecido la misma visibilidad que aquellas que se dirigen a las mujeres (Scott 2005a), lo cual es grave si consideramos que el estudio sobre el tema es decisivo para comprender mejor la propia constitución de la nación y del poder (Scott 2005b).

Se puede decir que la familia pasa a ser valorada como institución social relevante en la organización de las acciones públicas y en la propia idea de la democratización. De hecho, como intentaremos explicar, es un lugar estratégico para pensar no sólo en la trama de la sexualidad y la reproducción, sino sobre todo - lo que se torna evidente ahora, en el contexto posnacional (Martins 2004) —, en la trama de la normalización del orden social.

Si la familia era un tema menor en la organización de las instituciones sociales en general, obviamente se asumía como tema secundario en la organización de las instituciones públicas. Entretanto, el nuevo estatus sociológico adquirido por el asunto ha impactado en forma significativa a los estudiosos de las políticas públicas y a los planificadores y gestores, lo que en Brasil es evidente al estudiar las reformas en la salud pública. 
Por otro lado, se puede decir que la nueva comprensión de la familia va más allá de su definición como espacio prioritario de la sexualidad y la reproducción, para incluir las funciones morales básicas de la socialización y de la individualización, las cuales pasan a constituir un puente estratégico entre la comprensión sociológica tradicional de la familia y la nueva comprensión, como se puede concluir de ciertas investigaciones sobre el tema (Sarti 2005). Es decir, a pesar de que el lazo consanguíneo, la sexualidad y el trabajo continúen apareciendo como elementos relevantes en la caracterización de la familia moderna, tales elementos se muestran hoy insuficientes para explicar los nuevos desafíos de esta instancia en la producción, no sólo del 'mundo de la vida', sino también de las instituciones sociales en general y de aquellas políticas en particular. Varios estudios avanzan en esta dirección (Carvalho 2003).

Para que desarrollemos nuestras reflexiones sobre la actualidad política de la idea de familia, sin que nos perdamos en conjeturas abstractas, es importante relacionar la revisión del debate sobre familia con nuevas concepciones de políticas públicas, pues las remodelaciones conocidas por el grupo familiar siguen de cerca los cambios de concepciones sobre las intervenciones públicas y sobre la actuación de ciertos actores - gestores públicos, educadores, asistentes sociales y terapeutas familiaresen la concepción e implementación de políticas de control social. Esa intervención fue demostrada de forma convincente por Christopher Lasch cuando señaló que "la historia de la sociedad moderna es la afirmación del control social sobre las actividades antes relegadas a los individuos o a sus familias" (Lasch 1991:21); también lo reafirmó John Rodger (1996), para quien las políticas de control social nunca consideraron seriamente la importancia de un abordaje interactivo que valore al usuario o al cliente, revelando en el siglo XX un cierto sesgo autoritario.

Hay entonces dos tareas fundamentales para poder avanzar en una nueva concepción de política pública: una de ellas es representar el control social, la otra es repensar una noción de familia que ha servido de base para la acción gubernamental efectuada sola y/o apoyada por otros agentes públicos en el plano de las sociabilidades primarias.

En este texto intentaremos avanzar en algunos puntos de esta revisión, sabiendo desde luego que esta discusión tiene horizontes mucho mayores que no pueden ser alcanzados aquí, en la medida en que el debate tiene que ver con la propia reorganización del Estado en el contexto de la globalización.

Tal vez, lo que debe ser indicado como punto central es que la incorporación del elemento familia en el imaginario de la planificación pública trae consigo nuevos desafíos de revisión de la función redistribuidora típica del Estado (Rosanvallon 1981), una vez que la participación pasa a ser valorizada como pieza central en el juego político. Para Santos y Avritzer (2002:76), la valoración de la participación 
en el juego democrático nos obliga a reconsiderar el de la representación. Es el éxito de la articulación entre democracia representativa y democracia participativa, que "presupone el reconocimiento por parte del gobierno de que el procedimiento participativo, las formas públicas de monitoreo de los gobiernos y los procesos de deliberación pública pueden sustituir parte del proceso de representación y deliberación, tal como son concebidos en el modelo hegemónico de democracia" (Santos y Avritzer 2002). Y, complementamos, no se puede pensar la democracia participativa sin pensar la relación entre público y privado, y el lugar de la familia en la organización de la actividad pública.

La complejidad de una nueva política pública, abierta para el proceso participativo, puede ser presentada como expresión de un sistema de doble mano, o sea, conducido por dos lógicas: una es la familiar, generada por las relaciones cara a cara, que tiene su hábitat natural en el mundo de la vida cotidiana y es apenas influenciada indirectamente por las acciones generadas en el campo de la política; la otra lógica es la del control social, construida a partir de intervenciones organizadas de carácter administrativo, económico, asistencial, pedagógico, psicológico y moral, sobre el sistema de sociabilidades primarias, siendo influenciada directamente por las acciones políticas.

En cierta medida, la situación degradante de las familias pobres en Brasil, que tienen a las mujeres como jefes (Scott 2005a), resulta no de transformaciones realizadas de manera aleatoria en el interior del mundo de la vida - de la modernización o de reacciones espontáneas de los actores sociales directamente involucrados-, sino sobre todo de alteraciones estructurales entre el orden cultural, social y político; es decir, a partir de la interfase entre la vida cotidiana (espacio de saber simple) y las intenciones y prácticas generadas en el campo de las instituciones formales (espacio de saber científico y burocrático). En este sentido, Jelin (1994) tiene razón en decir que la familia y el mundo doméstico no son lugares cerrados, sino que se constituyen en relación a lo público:

los servicios, la legislación y los mecanismos de control social, así como aspectos más simbólicos como las visiones sobre el ámbito de aplicación de la medicina, las imágenes sociales prevalecientes sobre la familia y la normalidad, las ideologías e instituciones educativas, ayudan a definir en cada situación histórico-cultural, el ámbito de acción propio de la familia y la domesticidad. (Jelin 1994:1001)

Debemos reconocer que el análisis de las situaciones familiares hoy, indica que el futuro de esta institución depende más de las imbricaciones y desdoblamientos de las acciones, reacciones, estrategias, conflictos y alianzas forjadas en el seno de 
las sociedades mundializadas, a partir de experiencias asociativas y comunitarias localizadas, que de la 'voluntad de potencia' del grupo familiar propiamente dicho. Este reconocimiento es relevante para no culpar al grupo familiar como principal responsable moral de las dificultades enfrentadas en la conducción del proceso de socialización de los individuos. Por el contrario, es necesario entender que sin la familia la socialización queda comprometida y, por consiguiente, la propia expectativa de paz social.

Los estudios de antropología urbana ya demostraron, por ejemplo, la relevancia de las redes de parentesco en la realización de tareas ligadas a la manutención cotidiana de los miembros de la unidad doméstica (Jenin 1994). Pero esta comprensión aún no se ha transformado en orientación objetiva, en el nivel de las políticas públicas.

Tenemos, en definitiva, interés en demostrar en este texto que muchos segmentos de la sociedad organizada en general y de los intelectuales, líderes políticos, planificadores y gestores públicos, en particular, no se encuentran indiferentes a la importancia de repensar la política para adecuar los mecanismos e instrumentos de gestión a las nuevas exigencias de regulación y producción de la vida social en el plano local - lo que necesariamente lleva a revisar el lugar de las instituciones familiares. En Brasil, los esfuerzos de la reforma estatal prueban lo anterior. Estos esfuerzos persiguen recuperar la capacidad reguladora y redistributiva del Estado, que en las últimas décadas quedó muy comprometida con la expansión de la lógica mercantil y neoliberal (Rosavallon 1981, 1995), agravando las condiciones de sobrevivencia institucional y política de las sociedades nacionales.

Para analizar este esfuerzo de reforma del Estado con miras a la creación de nuevos mecanismos de promoción de la ciudadanía y de la participación popular en las decisiones políticas, fijaremos nuestra atención en el ejemplo de la salud. Esta representa, según nuestro entender, el intento más osado de reforma vía descentralización y territorialización de la acción pública, en busca de empoderar a las poblaciones locales y a la unidad familiar, incentivándolas a integrarse a la experiencia participativa y pública.

En otros textos tratamos la descentralización de forma general (Martins 2004a, 2004b, 2004c, 2004d); en este procuraremos enfocar este análisis prioritariamente en la crítica a la noción de familia-domicilio utilizada por los gestores públicos, de modo que podamos resaltar sus límites y potenciales. Según lo entendemos, esta noción tiene aspectos positivos y negativos que deben ser resaltados: por un lado constituye un intento de considerar seriamente la idea de familia en la elaboración de políticas públicas; por otro, la noción de familia-domicilio, como veremos, posee límites que pueden comprometer el avance de la reforma deseada.

A partir del enfoque de este complejo problema — la articulación de la política pública con la vida local y comunitaria, usando como referencia el núcleo 
familiar-, procuraremos avanzar en una crítica más sistemática de los límites de las políticas públicas, centrando nuestra atención en dos tareas: explicar ciertos aspectos relevantes del desarrollo del pensamiento sociológico sobre la familia para centrarnos en la crítica de los límites de la idea de familia-domicilio; y proponer la profundización de la comprensión de la familia como red social, dado que, sobre todo en las clases populares, que conocen servicios estatales muchas veces precarios, la red de parentesco aparece como más importante que los mecanismos formales en la solución de problemas de la cotidianidad (Jelin 1994).

\section{Nuevos sentidos de la acción pública a partir de la revaloración de la idea de familia}

Las dificultades para fundar en América Latina y en Brasil un Estado-proveedor ${ }^{1}$ están de alguna forma ligadas a la existencia de un poder-providencia tradicional, articulado en los municipios por un sistema de dominación oligárquico que organizaba mecanismos de control social fuera del Estado (Graham 1997). Esos sistemas locales crearon las condiciones para la reproducción de los grupos familiares de trabajadores a partir de una cultura de dependencia fuertemente marcada por el apadrinamiento y por las relaciones de parentesco de alianzas y de amistad. Así, a lo largo de los tiempos, los grupos sociales pobres se reprodujeron en las franjas de la sociedad organizada, dependiendo directamente de los propietarios de tierras para sobrevivir. Con frecuencia, el jefe local era —al mismo tiempo- propietario y representante del poder político, como lo demostraron estudios clásicos (Leal 1986, Queiroz 1976).

La existencia de una sociedad autoprovidencial que sustituía los márgenes de las grandes propiedades y bajo el control del poder oligárquico, constituyó en Brasil durante mucho tiempo — por lo menos hasta la primera mitad del siglo XX — la referencia de sobrevivencia de los segmentos sociales más humildes, que no tenían acceso a la protección social y pública ofrecida por un Estado-providencia. La imagen del poder público era - y eso sucede hasta la fecha-, ampliamente condicionada a figuras míticas (gobernadores, presidentes, diputados, alcaldes) que encarnaban el poder del gobierno y las poblaciones pobres permanecían sometidas a la lógica del mundo rural. A este destino apenas escapaban aquellas comunidades étnicas o religiosas que decidían fundar comunidades en las fronteras de las grandes

1 Uso el término providencia, traducido literalmente del francés etat-providence y que no pretende remeter al modelo social-demócrata europeo del Estado benefactor. 
propiedades, formando quilombos (comunidades cimarronas) y áreas 'libres' de pequeños propietarios.

Sucede que la precarización y la exclusión progresiva de esos segmentos sociales demuestra, según Inaiá Carvalho, que los sistemas de parentesco organizados a partir de esta lógica localista no-pública, en la segunda mitad del siglo XX perdieron progresivamente su capacidad de funcionar como amortiguadores de la exclusión y como mecanismos de protección de sus componentes, lo que llevó al deterioro de las condiciones de vida de la mayoría de la población (Carvalho 2003), y a la favelización de la pobreza en la periferia de los centros urbanos. Sucede que las políticas públicas no se modernizaron con la misma velocidad que se dio el incremento de los conflictos y demandas urbanas, lo que generó el deterioro de los servicios y el aumento de la insatisfacción popular.

El nuevo poder local, nacido de las presiones por la participación, viene organizándose con dificultades, muchas veces cooptado y/o corrompido por el poder tradicional (Santos y Ayrtzer 2005). De ahí surge la necesidad en el Estado de dirigirse a la vida municipal, a los sistemas comunitarios y a las esferas de la vida doméstica, para evitar que se amplíe la degradación de los sistemas familiares empobrecidos, como lo prueban investigaciones recientes (Scott 2005a).

Infelizmente, tales iniciativas todavía son tímidas. Pero la urgencia se torna incuestionable frente a la explosión de una violencia social que aumenta en forma simultánea las dificultades que enfrenta la red de parentesco para asegurar la inclusión social de sus miembros.

En esta perspectiva de reforma del Estado, con miras a responder a la intensa y caótica demanda social, la definición de la idea de familia a partir del contexto domiciliar — que fue adoptada recientemente en Brasil por el SUS (Sistema Único de Salud) y materializada por programas como el PSF (Programa de Salud Familiar) y el PSA (Programa de Salud Ambiental) — constituye tanto una innovación como una limitación en términos de metodologías de políticas públicas. ${ }^{2}$ Representa una idea innovadora al cuestionar en forma implícita la concepción — tradicionalmente

2 Mis reflexiones sobre la idea de familia-domicilio surgieron a lo largo del desarrollo de la investigación "Red de vigilancia, ciudadanía y problemas endémicos", de la cual fui uno de los realizadores y que contó con el apoyo del Ministerio de Salud y de la Facepe (Fundación de Apoyo al Desarrollo Científico y Tecnológico de Pernambuco), entre los años 2003 y 2004. El objetivo de esta pesquisa fue desarrollar un concepto de red de vigilancia que pudiese ser útil para el análisis y la evaluación de los programas de control del dengue, lo que está teoricamente explorado en un libro publicado sobre el asunto, titulado Redes sociais e saúde: novas possibilidades teóricas (Martins y Fontes 2004). A pesar de que el domicilio familiar no constituye un eje central de la planificación territorial del Programa de Salud Ambiental, observamos que él continúa siendo una noción indispensable para la ejecución del programa. 
dominante en la planificación estatal - de representar a las poblaciones que van a ser asistidas por los programas públicos, como un objeto-meta definido estadísticamente por el principio 'per capita'; ello, sin considerar el factor relacional, los niveles de pertenencia y de reciprocidad entre los individuos; sin considerar a los actores locales como responsables de todas las acciones que les conciernen directa $\mathrm{o}$ indirectamente.

Vemos, así, que independientemente de la definición de familia utilizada (biológica, cultural, funcional, etc.), la política pública tiende a apoyarse de modo progresivo en la idea del domicilio, para anclar la acción social en una esfera de actividad fundamental para la construcción de la esfera pública y de la ciudadanía.

La introducción de la noción de familia por el planificador público contribuye a deshacer la idea corriente de que el grupo familiar - al contrario del individuo- no constituiría un buen indicador de política pública, por estar asociado tradicionalmente a la esfera privada y doméstica. ${ }^{3}$ Vale decir, al representar la familia un factor arcaico, baluarte legítimo del orden conservador, no podría constituir un indicador confiable para pensar la modernización nacional.

Tal indicador debería ser engrosado por la figura del individuo aislado y egoísta que, en el auge del 'boom económico' de los años 70, aparecía como un modelo típico del ciudadano emancipado, que encaminaría su vida dejando de depender de las llamadas 'sociabilidades comunitarias y familiares' (Carvalho 2003:15). Últimamente, tal imagen del individuo desenraizado ha sido revisada a la luz de las críticas que demuestran el carácter ideológico de esta noción, su función para la lógica mercantil y su cara depredadora de lo social. Durante los años 80 y en particular en los 90, tal concepción individualista fue progresivamente revisada, abriendo las perspectivas de criticar las nociones individualizantes del grupo social.

La institución familiar fue progresivamente revalorada, observándose en la actualidad, de acuerdo con Maria de Fátima Sousa: "un gran número de propuestas de políticas sociales basadas en la concepción de ciudadano comunitario, que aproximan la asistencia institucional y no-institucional, la formal y la informal, incluyendo las redes de solidaridades primarias (parentescos, amigos, vecinos) y el voluntariado" (2001:23). En esa perspectiva — complementa la autora- la

3 El término familia se deriva de famulus (esclavo doméstico), vocablo que utilizaban los romanos para designar a aquellos grupos sociales surgidos entre las tribus latinas y caracterizados por la presencia de un jefe de familia que mantenía bajo control a la mujer, los hijos y esclavos, con poder de vida y muerte sobre el conjunto. En la Roma republicana, por otro lado, pertenecer a una familia significaba, fundamentalmente, pertenecer a un mismo grupo político formado por varias generaciones, al cual se tenía acceso por el nacimiento (hijos), por la adopción (esposa y extraños) o por la compra (esclavos) (Bilac 2003). 
estrategia de Salud Familiar en Brasil "fortalece el movimiento de descentralización de la salud, basada en la concepción de que las instituciones de salud deben ofrecer cuidados de atención básica en la comunidad, aproximándose a los usuarios" (Sousa 2001:23).

Entendemos, así, que la adopción de la idea de familia-domicilio como referencia metodológica para definir las acciones de salud localizadas territorialmente, tanto puede contribuir a emancipar el nuevo paradigma en salud, a la valoración de la ciudadanía participativa, como puede falsear el significado de las relaciones de parentesco y de pertenencias afectivas, económicas y culturales — presentes en la forma de redes primarias-, impidiendo que ocurra el empoderamiento deseado de los actores locales. La respuesta a este asunto es compleja y tiene implicaciones directas sobre la capacidad de programas territorializados que respondan efectivamente a los desafíos de la movilización cívica en favor de la esfera pública.

En caso de que este programa se burocratice y se limite a aparecer apenas como un esfuerzo de desconcentración del poder gubernamental federal, como objeto de luchas corporativistas indiferentes al sentido universal de la acción pública, su resultado será la diseminación de la incredibilidad de los usuarios con relación al interés real del gestor público en administrar el bien, con miras a la promoción de los derechos sociales y de la ciudadanía en el plano local. Si esta acción descentralizadora falla, necesariamente la desestabilización del sistema social se agrava.

Dicho esto, debemos ahora resaltar una cuestión que es de suma importancia para nuestra reflexión y que habla del cuestionamiento sobre el sentido sociológico y antropológico del término familia-domicilio, que está siendo adoptado por los planificadores y gestores públicos, a saber: ¿hasta qué punto el uso de la idea de familia, limitada al plano domiciliar, establece algún diálogo con la idea de familia como relaciones de parentesco que, según Levi-Strauss (2003), no debería limitarse a la unidad biológica, incorporando igualmente, más allá de los lazos de consanguinidad, los de alianza entre grupos? O, dicho de otra forma: ¿̨a idea de familia adoptada en esos programas constituye apenas un adjetivo explicativo del domicilio, es decir, la familia aparece como una noción de carácter funcional elegida con fines puramente pragmáticos y productivos? En definitiva, el privilegio atribuido a la idea de familia-domicilio, para la organización de programas territorializados de atención a la salud, ¿constituye o no una innovación conceptual en un contexto histórico-político de falla del antiguo Estado proveedor? ${ }^{3}$

4 Históricamente, el Estado brasileño, preso en las mallas del imaginario 'desenvolvimentista' (Martins 1992), revela crecientemente la incapacidad de comprender a la sociedad como un fenómeno mayor que aquel de los intereses de la economía de mercado, de comprender a la sociedad nacional como un fenómeno histórico formado no apenas por intereses económicos, sino igualmente por determinantes 
Deseamos observar pues, que, basándose en una perspectiva más amplia de familia, es posible que los programas de salud actúen en el sentido de derechos y obligaciones del ciudadano, a partir del plano local y comunitario, y al mismo tiempo contribuyan a activar las virtudes cívicas colectivas (volviendo a los individuos más participativos y comprometidos con su mundo social, en un contexto de exclusión social y de gran vulnerabilidad afectiva-emocional de las poblaciones pobres). El enfoque en la familia aparece aquí como estratégico para repensar la propia idea de lo local.

Es importante dejar en claro que, según nuestro entender, la introducción de la noción de familia-domicilio en la planificación pública nos parece un avance teórico evidente con relación a las concepciones tradicionales de políticas públicas, que no consideran las redes primarias, sino solamente los agregados estadísticos (la población es vista como unidad per capita sobre la cual se construyen agregados abstractos como, por ejemplo, niños sin vacunas, hombres enfermos, mujeres en estado prenatal).

Esta concepción es desconocida; aquí, niños, hombres y mujeres no son meras unidades estadísticas para ser objetos de cálculos y reglas impersonales, sino que por el contrario son unidades pensantes o 'átomos sociales', como decía Moreno (1987), fundador de la sociatría.

Pensar los agentes a partir de una red viva significa aceptar la existencia de una constelación social que los tome en cuenta estructuralmente, tanto a nivel de su existencia como en cuanto agrupación social, así como a nivel de la definición de su papel en la institución de la sociedad. Pero debemos ir más allá del explorar las perspectivas de avanzar en la crítica teórica de la familia-domicilio, para que entendamos sus límites en tanto operador institucional del trabajo de democratización de la acción pública.

Comencemos por el PSF. Su abordaje, como observa con propiedad Edilza Ribeiro, se centra sobre todo en el individuo dentro del domicilio familiar, sin fijarse claramente en la trama sistémica:

En este abordaje la familia se concretiza en la perspectiva del individuo, que es el foco real de atención, pudiendo ser la mujer, el niño, el portador de diabetes. O sea, la familia es el cliente nominal, pero no es el

culturales, morales, políticos y sociales. La visión economicista restrictiva del imaginario desenvolvimentista contribuye para producir anomalías sociales crecientes, al mismo tiempo que se revela como insuficiente para producir solidaridades colectivas que son básicas para la existencia de una sociedad nacional democrática. 
'objeto de cuidado'. Y, más aún, la familia tiene papeles para desempenar, tiene deberes y responsabilidades con el individuo enfocado y para ello necesita una orientación de entrenamiento. La familia también se entiende como aquella que interfiere positiva o negativamente en el proceso de salud/enfermedad del individuo. Dada la expectativa de deberes, las condiciones reales de capacidad de cuidado de la familia, vistas de forma multidimensional, son frecuentemente olvidadas, además de ser objeto de juicios de valor por parte de la comunidad y de profesionales. (Ribeiro 2004:11)

La definición de familia a partir del domicilio constituye una de las descripciones posibles de esta noción. Es importante observar que tal concepción apenas rompe parcialmente con la perspectiva individualista que inspiran los programas de salud, que valoran la dimensión familiar. Nótese, igualmente, que tal noción termina por exagerar el valor del domicilio físico, en detrimento de las tramas interpersonales y de las imposiciones propias del grupo primario. $\mathrm{O}$ sea:
El ambiente, la infraestructura material de la familia, las condiciones de cuidado de ese ambiente, son el foco de la atención. Lo sustitutivo, lo que toma el lugar de, o representa a la familia es, pues, su espacio o con- texto físico. El domicilio es la base del lanzamiento de datos estadísticos y epidemiológicos del proceso salud/enfermedad, de la caracterización de la familias que tienen condiciones y de aquellas que necesitan ayuda, de aquellas que requieren vigilancia de salud, o de otras que están en situación de riesgo socio-ambiental. (Ribeiro 2004:41)

Sin duda, al introducirse la noción de familia domiciliar (aun cuando limitada al factor territorial) en lugar del factor per capita, que es muy abstracto, los que formulan y planifican los programas territorializados produjeron un cambio cualitativo relevante en el nivel de focalización de la acción pública: la población pasa de la condición de objeto-meta a la de sujeto-meta. Pero, según nuestro entender, la principal dificultad conocida por esas acciones, para avanzar en el proceso de descentralización y democratización, continúa siendo engrosada, no por cuestiones burocráticas, administrativas o financieras, sino por las limitaciones metodológicas de la noción de familia utilizada para organizar territorialmente a la 'población-meta'. O sea, estos cambios de visiones sobre los fundamentos de la vida asociativa por la gestión pública son aún insuficientes para apoyarse en una reforma efectiva del Estado. La noción de familia-domicilio, como se observa, padece de dos limitaciones: una de ellas respecto del énfasis sobre el individuo en 
detrimento de las interacciones sistémicas; la otra es el énfasis sobre el ambiente físico en detrimento del orden simbólico.

Para que avancemos en el debate importa establecer, entonces, la separación entre una visión conservadora y una visión moderna de familia. Si la primera, la conservadora, está de hecho históricamente vinculada a la reproducción de mecanismos de poder y de control patriarcales y autoritarios, la segunda, como intentamos demostrar, representa una posibilidad efectiva de pensar el surgimiento de una esfera pública democrática en el plano local. Claro, esta división contiene diversas subdivisiones, pero para nuestro raciocinio con respecto a las condiciones de organización de un nuevo pensamiento político y administrativo hegemónico en la política en el seno del Estado, ella es suficiente.

Según la perspectiva conservadora, podemos avanzar en la hipótesis de que la noción de familia-domicilio no rompe con la visión autoritaria de familia tradicional (Martins 2002), en la medida en que el poder de decisión sobre lo que es bueno o no para la comunidad queda dependiendo, en gran medida, de la voluntad del Estado y de los políticos conservadores, que por todos los medios intentan cooptar y someter la democracia participativa (Santos y Ayritzer 2002). Para defender la posibilidad de una noción de familia que refuerce el carácter democrático deseado, necesitamos avanzar por otra vía: ni la de la familia biológica ni la de la familia-fuerza de trabajo ni, tampoco, la de la familia-domicilio. Debemos, por el contrario, estimular una concepción que en primer lugar valore los sistemas de pertenencia, que valore positivamente el vínculo afectivo entre personas próximas en la constitución de asociaciones libres y espontáneas en el plano local (entre personas próximas por lazos de consanguinidad, vecindad o amistad). Finalmente, debemos superar los límites de la idea de familia-domicilio para avanzar en una comprensión relacional de la familia, que dé cuenta de las imposiciones simbólicas y materiales, individuales y grupales. Para ello, buscaremos apoyo en conceptos tales como reciprocidad, solidaridad, confianza y dádiva, de modo que edifiquemos una noción de familia que haga énfasis en el valor de las redes sociales (Martins y Fontes 2004) y en la asociación democrática (Chanial 2004).

Para explorar las posibilidades de democratización de la política pública a partir de los cambios en curso en la salud, necesitamos organizar una agenda de reflexión que abarque algunos temas centrales. En primer lugar, comprender el cambio de estatus de la familia en los estudios sociológicos y verificar cómo este factor puede servir para profundizar en la comprensión de la familia; en segundo, entender el acuerdo familiar como una red simbólica y material al mismo tiempo que aparece como factor decisivo para la transformación del 'mundo de la vida' en base real para la formación de una existencia asociativa y participativa en el plano local. 


\section{Revisando el estatus sociológico de la familia}

Tradicionalmente, la sociología encara a la familia como uno de los grupos sociales relevantes en su búsqueda de explicar las razones por las que surgen los agregados sociales. Para Emile Durkheim, dentro de esos grupos la familia se destaca de manera especial por el hecho de ser el agregado más simple y el más antiguo, teniendo funciones para la sociedad que no podrían ser explicadas ni por la psicología ni por la biología. Puede decirse que el fundador de la escuela sociológica francesa fue el primero en proponer una visión de la familia basada en las ideas del matrimonio y de la alianza, apartándose de otras proposiciones que definían el grupo familiar en las perspectivas del determinismo biológico o de la psicología de los sentimientos (Durkheim 1975).

El fundador de la escuela sociológica francesa consideraba su perspectiva moral como fundamental para el desarrollo del método comparativo, que deseaba incorporar en el estudio de sistemas familiares diferentes, y en la comprensión del paso de una sociedad basada en la solidaridad mecánica a otra orgánica. ${ }^{5} \mathrm{O}$ sea, al mismo tiempo que defendía la importancia de un abordaje plural de la familia, Durkheim creía que la familia moderna europea era el resultado de un lento desarrollo de la sociedad. En este sentido, el pensamiento dominante hasta hace poco tiempo de que la familia nuclear sería un fenómeno ontológicamente superior a los acuerdos familiares tradicionales y de naturaleza irreversible está inspirado, en cierta forma, en este ideal de progreso social de la familia, heredado de la escuela francesa de sociología.

Más de cien años después de esta primera sistematización de los estudios de la sociología de la familia, constatamos que el acuerdo nuclear urbano (formado básicamente por la composición de padre y madre biológicos e hijos/as) no se afirmó como un patrón universal superior que se impondría históricamente sobre los

5 Emile Durkheim pronunció una conferencia inagural de ciencias sociales en la Universidad de Bordeau en 1888 titulada "Introducción a la sociología de la familia", en la cual procuró explicar la complejidad de la familia moderna y su importancia para comprender la solidaridad orgánica en la sociedad industrial. El fundador de la sociología francesa propuso a los oyentes la siguiente cuestión: “¿Qué puede ser más interesante que observar que la vida moderna, que parece ser tan simple en apariencia, se revela mediante una variedad de elementos y de relaciones estrechamente imbricadas unas a las otras y que conoce en la historia un lento desarrollo durante el cual estos elementos sucesivamente se formaron y se combinaron?" (Car qu'y a-t-il de plus intéressant que de voir cette vie de la famille moderne si simple en appparence, se résoudre en une multitude d'éléments et de rapports étroitement enchevêtrés les uns dans les autres et de suivre dans l'histoire le lent développement au cours duquel ils se sont successivement formés et combinés?) (Durkheim 1888). 
sistemas de pertenencia heredados de las antiguas familias domésticas (formadas por grandes agrupamientos de parentesco, de dependencia y fidelidad). La experiencia del siglo XX nos muestra, igualmente, que este modelo nuclear no posee la característica de aparecer como un vector naturalmente generador de solidaridades colectivas y de una cultura de esclarecimiento universal.

Por un lado, se nota que este modelo de familia nuclear moderno no se universalizó, como podrían suponer aquellos teóricos que asocian la estructura de la familia al desarrollo de la sociedad industrial. Al analizar el modo de organización de los sistemas familiares en las sociedades donde la industrialización urbana y el mercado de trabajo especializado no se tornaron fenómenos hegemónicos en la organización de la vida social y donde el Estado-providencia tiene eficiencia limitada, se nota que las redes de parentesco — y no la familia nuclear-, respondieron por las tareas básicas de la cotidianidad (Jelin 1994). Aun en los países más avanzados, que defienden un modelo republicano laico, se observa que los inmigrantes reproducen modelos comunitarios inspirados en múltiples tradiciones religiosas, étnicas y culturales, que frecuentemente contradijeron los principios legos. El debate reciente sobre el uso o no del velo en las escuelas francesas por parte de mujeres jóvenes musulmanas es un ejemplo de ello.

Por otro lado, de algunos años para acá se puede realmente decir que está en curso una corrosión de los lazos sociales que afecta de manera creciente las condiciones de existencia de la institución familiar como grupo básico de socialización, y no sólo aquel modelo de la familia nuclear. Si este trabajo de corrosión se debe en parte a la dinámica depredadora de la acumulación capitalista que genera ondas crecientes de exclusión social, no se puede eximir al Estado y las políticas públicas de tener parte de la responsabilidad en la degradación del sistema social. Tales políticas públicas contribuyeron para ampliar la desigualdad, la falta de respeto y la exclusión que se revelaron con intensidad, al desmontarse los sistemas sociales primarios (Sennet 2004).

El sentido común interpretaba, hasta la década de los 90 , esas modificaciones del sistema familiar como 'crisis de generaciones' que sería administrada naturalmente por el tiempo. Pero los hechos no avanzan dentro de esa lógica de progreso social administrable. La degradación de las condiciones de vida y el aumento de la exclusión social han afectado las bases de la solidaridad social, contribuyendo a diseminar una cultura de violencia que afecta directamente al grupo doméstico (Morroson y Biehl 2004). Esto nos pone ante el desafío de realizar una revisión sociológica necesaria de lo que es la propia idea de pobreza (Schwartzman 2004).

Se puede, por consiguiente, avanzar en la hipótesis de que el desmoronamiento de la institución familiar moderna es un proceso paradójico con aspectos positivos y negativos. Así como es expresión de las nuevas exigencias de adaptación de 
los individuos a la sociedad, revela también el impacto corrosivo de la violencia social. Por una parte, tal proceso de desmoronamiento del patriarcado sugiere perspectivas esperanzadoras en términos de mayor democratización de la sexualidad, desprendida de la mera procreación (Bozon 2004) y valora la intimidad (Giddens 2003). Por otra parte, tenemos que reconocer que la noción sociológica clásica de familia - que se refiere al mundo del trabajo y al modo como fue pensado lo social en la modernidad - pierde su aura 'progresista' en un contexto de crisis de la protección social, de pérdida de solidaridad y de exclusión creciente (Rosavallon 1981, Castel 1995, Cavalcanti y Burity 2002), alcanzando principalmente a los grupos más humildes. Esta degradación ha sido constante y la ascensión de la mujer a la condición de jefe de familia no interrumpió el proceso, como observó Parry Scott (2005a), al estudiar a familias de ingresos bajos en la ciudad de Recife.

La noción tradicional de familia es, pues, insuficiente para revelar la nueva dinámica de las pertenencias, de los conflictos y alianzas, resultante de la crisis del grupo familiar doméstico de inspiración paternal. Nuevos e inéditos acuerdos conyugales y domiciliares apuntan hacia la redefinición del poder doméstico en todos los planos sociales, a partir de las tensiones entre, por una parte, las dificultades de la familia de reproducir el sistema; por otra, las presiones de autonomía vividas por los miembros individualmente a partir de otras redes de las cuales forman parte (amigos, colegas de trabajo, compañeros de acción sindical y/o política, etc.). Por otro lado, nada comprueba que la ecualización de los poderes entre hombres y mujeres, proveniente de los avances del movimiento feminista (Heilborn 2004b) condicione la manutención del modelo de la familia nuclear urbana a ser lo más interesante para asegurar la democratización de lo cotidiano. Al contrario. Con mucha frecuencia se observa que son las redes de parentesco o de parentela, construidas por un número significativo de individuos casi-parientes (padrastros, madrastras, entenados, entenadas), de nuevos dependientes y amigos, las que vienen creciendo, abriendo un abanico de formación diversificada de nuevos acuerdos familiares que modelan esta nueva distribución de poderes.

Naturalmente, crecen las tensiones sexuales, étnicas y culturales, las que llevan a los individuos a cuestionar los antiguos modelos de relación (Giddens 1993) y a adoptar nuevos patrones de sexualidad y reproducción, así como nuevas modalidades de poder en el sistema familiar capaces de atender las presiones de participación en el interior de los nuevos acuerdos familiares (Bozon 2004, Heilborn 2004a). En suma, junto con la redefinición de poderes, de identidades y de lugares, esta diferenciación creciente de formas de poder y de diversificación de las 'jefaturas' de los acuerdos domésticos (madre jefe de familia, padre jefe de familia, madre y padre que permanecen ambos jefes de familia, tío jefe de familia, etc.) tiene impacto necesariamente sobre la esfera extradoméstica, aquella de las redes de vecindad, 
amistad y asociación. O sea, a la par de la reorganización interna del poder doméstico, crece la presencia de una cultura comunitaria y de vecindad que refuerza la perspectiva de una esfera pública asociativa. Así, por ejemplo, para encarar el cúmulo de responsabilidades es común que madres-vecinas se presten servicios de ayuda mutua (como cuidar hijos, prestar alimentos o dinero, etc.).

En este contexto de cambio social intenso y de revisión de presupuestos teóricos, la familia deja de ser vista como mera expresión de la producción material de la sociedad, para aparecer como mecanismo decisivo en la reproducción de la vida social y como favorecedora de reciprocidades igualitarias. Esta tendencia se observa, sobre todo, en el seno de las clases medias (Heilborn 2004b), sin embargo, puede proponerse que, a pesar de las condiciones de vida degradantes de los grupos empobrecidos, el empoderamiento de la mujer es un hecho incuestionable (Scott 2005b). Siendo así, podemos sugerir que está en curso la formulación de una nueva institución social familiar que aparece con el diseño de una red social primaria y abierta, o sea, como un sistema de reciprocidades y de pertenencias que involucra, en principio, a individuos vinculados a los mismos grupos consanguíneos o culturales (esto explica su carácter primario), pero que tiende a extrapolar al grupo original para incluir a otros individuos no necesariamente vinculados por lazos de consanguinidad (de ahí el carácter abierto de esta red que tiende a cruzarse con las redes de vecindad, de asociación y de usuarios de servicios públicos como es el caso, por ejemplo, de los grupos de hipertensos o de diabéticos que se reúnen bajo el incentivo de las unidades públicas de salud).

Maurice Godelier (2004) introduce una clasificación del sistema de parentela actual que nos parece pertinente para nuestras reflexiones sobre este nuevo modelo de familiares, que es, a su vez, la matriz estructurante de una posibilidad amplia de nuevos acuerdos asociativos. Según este autor, el sistema se compone de tres elementos que se combinan entre sí, constituyendo una estructura profunda de parentela, a saber: la familia nuclear, la red de familias emparentadas por lazos de consanguinidad o alianza y la parentela strictus sensus, entendida a partir de un doble registro: como red de parientes (del individuo) y como red centrada en el individuo. Esta tercera variación surge en el momento en que el individuo (reflexivo) aparece como punto de partida para el surgimiento de otra parentela. Se puede hablar aquí de 'individuo-eslabón', de un agente que adquiere la autonomía reflexiva, de un ser que se posiciona críticamente contra la fuerza coercitiva del grupo y que pondera en forma reflexiva sus pulsiones y acciones. Se trata, por cierto, de un fenómeno moderno no observado en sociedades tradicionales en la medida en que en esas últimas la personalidad individual, este 'yo', es siempre una expresión cultural y psicológica frágil, que no sobrevive fácilmente fuera de la conciencia colectiva (Mauss 2003a). 
Así, tendríamos como desdoblamiento de este tercer elemento dos redes de parentela: una de ellas sería aquella representada por la red familiar; la otra, aquella formada por individuos emparentados a partir de una combinación abierta que involucra vínculos tradicionales y postradicionales. Se trata, como dice Godelier: "de redes abiertas cuyos límites dependen de múltiples factores que nada tienen que ver con la parentela: proximidad espacial de las familias y de los individuos, cambio de estatus social de algunos de ellos o de algunos que no se frecuentan más, desapariciones causadas por epidemias, guerras, etc.” (2004:12).

Con respecto a lo que se dice en particular sobre la familia occidental, Godelier nos recuerda que la misma ha sufrido cambios profundos debido a tres tipos de factores: la relación de los individuos con la sexualidad, los lugares respectivos de los hombres y mujeres en la sociedad y la posición de los niños (Godelier 2004). Esos factores han contribuido al crecimiento de 'familias-recompuestas', resultantes de separaciones, divorcios y nuevos enlaces. Como resultado de ello, se observa el surgimiento de diversos nuevos acuerdos familiares, que implican tanto situaciones de parentesco clásico (biológico y social) como de casi-parentesco, en las cuales se hacen presentes nuevos acompañantes de los padres y nuevos hijos adoptados (Godelier 2004). En suma, hay una negociación intensa de cuerpos, hablas, gestos e intenciones que funcionan como matrices simbólicas permanentemente accionadas en la producción de nuevas modalidades de organización social.

Para designar a la familia en esta perspectiva hermenéutica arriba apuntada, el término acuerdo familiar nos parece apropiado, en la medida en que permite observar la dinámica particular de organización de esos grupos primarios en diferentes contextos históricos, culturales y sociales. El acuerdo - una expresión singularizada de redes de parentesco y de redes de parentela, que son más amplias- permite que la estructura de la red se adapte a diversos contextos históricos y culturales, tanto moldeando la acción grupal como adaptando a esta a las exigencias de personalización de sus miembros. La negociación de nuevos acuerdos permite observar la presencia de una compleja vinculación asociativa y un creciente proceso de diferenciación destacado por la individualización en la familia. Pero, como lo dicen Thumala y Arnold-Cathalifaud, "la individualización no contradice la presencia de acciones colaborativas que pueden tener un impacto que empalma con las nociones de transformación sociales" (2006:3).

En fin, las nociones de red y de acuerdo familiar son convincentes para discutir con mayor profundidad dos aspectos: uno de ellos, los límites de la noción de familia-domicilio que está siendo adoptada por los programas de salud en Brasil; el segundo, la presencia de una lógica de capilaridad que se forma espontáneamente y responde a la necesidad de reproducción, sexualidad y socialización de las agrupaciones humanas. 


\section{Descentralización estatal, política pública y acuerdo familiar}

Los cambios en la salud son ejemplares para exponer los límites de la descentralización y los desafíos crecientes conocidos por los gestores públicos; asimismo, permiten dar cuenta tanto de la heterogeneidad de las situaciones sociales del mundo de la vida, como de la insuficiencia de la matriz teórico-metodológica utilizada para asegurar la redistribución de los bienes públicos en este plano de las socializaciones primarias.

Se reconoce que antes la política pública era más simple por el hecho de que el 'público' al cual se dirigía respondía a cierto patrón cultural uniforme: el trabajador vinculado al mercado formal de trabajo que cotizaba para mantener el fondo público el cual, a su vez, era dirigido a políticas públicas para ese mismo asalariado, con lo que se aseguraba la lógica productiva y reproductiva de la sociedad industrial. Por otra parte, la presión sobre la acción estatal era limitada por modelos de sociedades agrarias, es decir, que mantenían a gran parte de sus poblaciones en el campo, de manera que se reducía la presión política de los trabajadores sobre la gestión pública.

Ahora, con la disminución del número de individuos que cotizan, por un lado, y con el aumento de la exclusión social en las ciudades, por otro, la capacidad del Estado de responder a las nuevas demandas a partir de una planificación centralizada, se torna crecientemente improductiva e ineficaz sobre todo cuando este Estado — como sucede en el caso de Brasil- obedece tradicionalmente a una lógica oligárquica poco tolerante respecto de la presión popular por la participación. En este sentido, la descentralización del poder central, con miras a movilizar los poderes y voluntades locales y permitir la institucionalización de nuevos mecanismos de participación, como los consejos y los foros, es la única salida para que el Estado se adapte a la nueva demanda por participación y reconocimiento, que nace de grupos de individuos que nunca entraron en el mercado formal (Pochamann y Amorim 2003), y para favorecer la democratización de la acción pública.

La nueva planificación pública en la salud debe ser entendida, por consiguiente, como una adaptación institucional necesaria para contener la amenaza del caos social, derivada de un sistema político que busca ejercer históricamente un estrecho control sobre los ciudadanos. La implementación de acciones descentralizadas de redistribución de recursos y de responsabilidades de acciones y de servicios con fortalecimiento del plano municipal pero, sobre todo, del plano comunitario y asociativo, aparece como una solución imperativa. Se parte de la idea de que cuanto más cerca quede la decisión del hecho, más legítima será la decisión a ser tomada y más posibilidades habrá de acertar. Para ello se hace menester crear mecanismos e instrumentos que motiven a las poblaciones locales a participar de las iniciativas 
de interés común, condición necesaria para el surgimiento de una esfera pública democrática.

O sea, nuestra intención es demostrar que el avance de programas que se refieren a la familia (como es el caso de aquellos dirigidos a la salud en Brasil) para promover la democratización de decisiones y de apoyo a la participación efectiva de la población en la creación de una ciudadanía activa, si bien es importante aún es precario. Su sustenibilidad, a mediano y largo plazo, depende directamente, según nuestro entender, de la posibilidad de que las políticas públicas adopten una nueva perspectiva teórica, por la cual el usuario no sólo sea visto como un 'público-meta', sino también como un coautor de la acción pública.

El paso de un entendimiento del usuario como 'público-meta' a 'sujeto-meta' no puede ser obtenido por una mera operación administrativa o jurídica. Se hace necesario abandonar una posición funcional del usuario (engranaje pasivo de la acción de planificación) para adoptar una comprensión activa y política de este usuario. Tal comprensión nos es sugerida por la noción de público del filósofo norteamericano John Dewey (1997). Para él, el público involucra a todos aquellos que son afectados por las consecuencias de ciertas acciones que ultrapasan a los directamente involucrados, llevando a que se valore una experiencia de interés común que es superior a los intereses individuales (Dewey 1997). Según nuestro entender, esta concepción de Dewey de público puede ser útil para concebir al usuario en tanto sujeto-meta, como expresión viva del público. $\mathrm{O}$ sea, tal cambio de perspectiva no depende únicamente de la adopción de métodos estadísticos más avanzados, como ya fue dicho, sino de un método comprensivo que revele la presencia de un sujeto supraindividual, aun cuando tal sujeto no tenga conciencia de su poder real. De hecho, la 'voluntad de potencia' de los actores locales es inhibida debido a que los miembros de la comunidad son frecuentemente prisioneros de enredos sistémicos complejos (Hellinger 1999). Es decir, la comprensión del usuario como público es obstruida fácilmente por los conflictos que atraviesan sus redes de relaciones, sean de parentesco o de socialización. Esto perjudica, asimismo, el entendimiento de los conflictos psíquicos y culturales subyacentes al tejido social, los cuales son fenómenos relevantes para la producción de alianzas y pactos asociativos.

Nuestro propósito en este texto es demostrar que, a pesar de los avances importantes obtenidos con la implementación del Sistema Único de Salud (SUS) en los últimos 15 años, en Brasil, el proceso descentralizador con miras al empoderamiento de la sociedad civil parece encontrar obstáculos crecientes para su viabilidad. Las dificultades que tienen los agentes sociales para integrar la lógica de la acción democrática (redistribución de responsabilidades e incremento de la participación) y los principios de la acción descentralizadora (universalidad, integridad e interdisciplinariedad), revelan la confusión existente en la definición conceptual 
del 'público-usuario'. Tales obstrucciones revelan también los límites teóricos y metodológicos subyacentes a la idea de público-meta.

La noción de público-meta utilizada en esos programas constituye —según nuestro entender y como se dijo antes, pero nada cuesta repetirlo — una categoría de fácil manejo estadístico, pero que reduce enormemente la complejidad de la trama social. Tales programas aún se apoyan sobre una noción abstracta de individuo (conveniente para la organización del trabajo y de los modelos de protección social en el capitalismo industrial), insuficiente para atender la complejidad sistémica de la sociedad civil. Este modelo estadístico puede responder satisfactoriamente a las hipótesis casuales producidas por el planificador para definir la política pública (nivel de renta, género, condiciones de vivienda y de salud, entre otras), pero responde de manera muy insatisfactoria a la condición dialógica exigida por la trama de la descentralización, con la exigencia de participación y de formación de una esfera pública activa.

Semejante limitación puede ser entendida a partir de algunos puntos: a la complejidad de los acuerdos familiares, que sirven como soporte para formatear la idea de público-usuario, no se puede tener acceso por modelos estadísticos; la movilización de los actores locales, en busca de la definición de modelos dinámicos y participativos, no puede ser resultado de la mera voluntad del gestor estatal. Semejante movilización debe resultar, no de un poder central superior, sino de la valoración de la experiencia y de la libertad de la voluntad humana, como enfatiza el filósofo pragmatista norteamericano William James (1981), y de la existencia de mecanismos de trueque (Mauss 2003) y de reciprocidades (Simmel 1939) que favorezcan la experiencia del bien común como resultante del esfuerzo colectivo y supraindividual.

El modelo funcional del 'público-meta' aparece, para ser más claros, como un instrumento de deshumanización de las políticas públicas (Martins 2003), en la medida en que no considera las tramas subjetivas y las conexiones subterráneas de la vida social local, haciendo énfasis en indicadores superficiales e inadecuados para detectar el cambio social.

Por otro lado, las posibilidades de superación de los límites de este método funcional, adoptado por la planificación oficial, dependen directamente de la opción de explorar el potencial sistémico y complejo de las redes sociales primarias, que atraviesa lo que genérica y abstractamente designamos como familia. De hecho, más allá del exceso de abstracción de esta noción clásica de la antropología que constituye la familia, lo que verificamos en el mundo concreto son tejados de relaciones creadas por la experiencia vivida, que sugieren constituir las relaciones entre grupos de individuos — que viven en proximidad física y/o emocionalcomo una 'ecología profunda' (Capra 1998). Vale decir, la idea de familia como red social sugiere posibilidades teóricas inéditas para entender los rumbos que 
la política pública debe adoptar, con miras a apoyar la creación de movimientos de solidaridad a partir de programas territorializados (que trasciendan el ámbito familiar y favorezcan la vida asociativa y comunitaria).

\section{Familia como red social: una salida para la descentralización}

Como ya dijimos, es necesario dar un nuevo significado a la noción de familia, de modo que superemos la visión estrecha de la familia-domicilio, en pos de que integremos una visión relacional de familia-red. Esta visión más amplia sólo puede ser apreciada en su complejidad si comprendemos la familia a partir de un doble enfoque crítico: el primero, el de la teoría de la red, permite visualizar el grupo familiar no como una suma de unidades sino como una totalidad que se distribuye y se afirma por sus miembros; el otro, el de la teoría de la dádiva, permite entender la lógica propia del vínculo social, que es la de las obligaciones mutuas generadas por acciones de donación, recepción y retribución (Mauss 2003) ${ }^{6}$

Avancemos en algunos elementos para intentar aclarar esta base conceptual, antes alertando al lector de que esta tarea constituye un desafío aparte que sólo puede ser parcialmente respondido en este artículo.

Entiéndase red social aquí no sólo como instrumento o método de análisis auxiliar, como aparece con frecuencia entre los norteamericanos que trabajan con la idea de network analysis (Wasserman y Faust 1994), sino como una teoría compleja que se estructura progresivamente a lo largo de la modernidad en el siglo XX, con miras a explicar la dinámica relacional del hecho social (Martins 2004).

Cuando las ciencias sociales se apropian de la noción de red - como aclara por su parte Pierre Mercklé- es para poder dislocar el análisis sobre los individuos y sus atributos para "las relaciones que ellos establecen entre sí, para la comprensión de las formas que toman esas relaciones y cómo ellas contribuyen a modelar los comportamientos individuales y las estructuras sociales" (Merklé 2004:6). En una dirección paralela, Breno Fontes aclara que la noción de redes sociales tiene un papel destacado para revisar el capital social que los individuos poseen y para tener acceso a sus recursos: "el diseño de las redes sociales permitiría a los actores

6 No es posible explicar aquí la teoría de la dádiva. Para quienes consideran necesaria mayor información sobre el asunto, existe en portugués un material adecuado: el propio texto de Marcel Mauss, "Ensaio sobre a dádiva" (citado en la bibliografía); el texto de Jacques Godbout escrito en colaboración con Alain Caillé, O espírito da dádiva (1998); el libro de Alain Caillé titulado Antropologia do dom: o terceiro paradigma (2002), y la antología organizada por Paulo Henrique Martins cuyo título es $A$ dádiva entre os modernos: discussão sobre os fundamentos e as regras do social (2002). 
sociales, por lo tanto, formar un stock de capital social con el fin de movilizar recursos" (Fontes 2004:49). O sea, además de enfocar el vínculo interpersonal, la red también permite revelar los recursos materiales y simbólicos (confianza, amistad, etc.) que los miembros del grupo disponen para hacer girar la 'rueda de la vida' comunitaria, ya sea esta un grupo primario como la familia o un grupo secundario como las asociaciones formales.

$\mathrm{Al}$ procurar en el presente texto valorar teóricamente el acuerdo familiar como un factor social en forma de red, buscamos demostrar que, a pesar de su característica jerárquica típica, la familia persiste en aparecer como un referente socioantropológico central para pensar la ciudadanía democrática en el plano local y en el plano de las políticas públicas y sociales. Limitada a una estructura estática (el modelo de la unidad biológica), a un acuerdo social territorial o a una imagen abstracta de un 'público-meta', la familia tiene apenas interés estadístico, burocrático y económico.

Por el contrario, entendida como una red, como un acuerdo social en movimiento (y que varía según las diferencias históricas y culturales de cada sociedad), la familia puede aparecer como un operador simbólico decisivo en el paso de la esfera privada a la esfera pública. En tanto operador simbólico, la familia debe ser vista sobre todo como una red de relaciones primarias en la cual se concibe la reproducción física e institucional primera de la sociedad y en la que se esboza la socialización de los actores sociales. ${ }^{7}$ En esta perspectiva, esta se manifiesta —nos dice Alain Caillé - como 'una sociabilidad primaria privada', cuya presencia, como tal, es fundamental para el surgimiento de una 'sociabilidad primaria pública' que toma la forma de asociaciones civiles (Caillé 2004b).

7 La familia puede constituir una red de solidaridad inspiradora del pluralismo y de la participación social y pública, siempre y cuando la consideremos como una red social primaria básica para movilizar deseos colectivos y viabilizar alianzas locales, sobre las cuales puede prosperar la idea de público. Tal perspectiva democrática de familia aparece, en principio, como una contradicción, dado que la familia biológica constituye un sistema jerárquico por excelencia y no un sistema igualitario. Pero la noción biologizadora de este sistema primario es limitada, sin lograr abarcar las implicaciones sociológicas, jurídicas, culturales y sociales del fenómeno. En esta perspectiva más amplia, Margareth Ângelo y Regina Bousso proponen definir la familia como un sistema o una unidad cuyos miembros pueden o no estar relacionados o vivir juntos, puede contener o no niños, de un único padre o no. "En ella existe un compromiso y un vínculo entre sus miembros y las funciones de cuidado de la unidad consisten en protección, alimentación y socialización" (Ângelo e Bousso 2001:13). Las autoras señalan que en relación al aspecto operacional "la definición debe también considerar las creencias de la familia sobre su concepción de familia. De esa forma, es fundamental preguntar a qué la familia considera familia, aunque el PSF solamente registre los componentes de familias que residan en el area contemplada" (Ângelo e Bousso 2001:14). 
La familia-red presenta una perspectiva sociológica-antropológica más amplia, y explica un conjunto de lazos y pertenencias — a veces oculto, a veces explícito- a través del que se producen las identificaciones de lugares, de dádivas y de reconocimientos, sin los cuales no es posible concebir los fundamentos de la ciudadanía y ni siquiera los del ser humano en tanto sujeto social. La comprensión de la familia como red social puede ser mejor aclarada por el siguiente comentario de Alain Caillé, al respecto de cómo se procesa a partir de la dádiva el reconocimiento del otro en cuanto protagonista y compañero (siendo la dádiva la condición primera para resolver conflictos y forjar alianzas):

De la misma forma que el reconocimiento físico, la identificación —el hecho de conocer nuevamente lo que ya fue reconocido- queda subordinado al reconocimiento valorativo, aquel que marca a alguien o algo, como portador de sentidos y revelador de afectos. De esa misma forma se puede pensar que el reconocimiento valorativo se reporta en definitiva en el reconocimiento, en el sentido de gratitud. Gratitud con el acto de donación gratuita o de un regalo inesperado. (Caillé 2004a:16)

El núcleo familiar aparece, en la perspectiva de dádiva (Mauss 2003) y de red, como un sistema de reciprocidad que tiene base moral en el valor-confianza (generando las condiciones de proximidad e intimidad), en la identificación y reconocimiento de lugares (posibilitando la idea de actor social) y en la autoayuda entre los miembros de la comunidad primaria, como una especie de círculo virtuoso que posibilita acciones políticas y alianzas bien logradas en el plano intrapersonal y en el plano interpersonal (Chanial 2004, Caillé 2004b). Sylvia Mello (2003) propone tres tipos de planos de construcción de la familia-red: familia nuclear propia, familia compuesta de varias familias nucleares que por cuestión de sobrevivencia habitan juntas y, por último, familia que incluye parientes de parientes y compadres sin lazos consanguíneos. Entendemos que tal concepción amplia de familia distribuida en tres círculos puede contribuir a explorar aspectos sutiles de la organización de la lógica asociativa y del estímulo a la ciudadanía, como el sentido de responsabilidad pública y la participación de los individuos en redes en los proyectos colectivos. La resolución de conflictos y el establecimiento de alianzas en esos diversos planos constituyen etapas decisivas para que los programas públicos puedan alcanzar los sistemas primarios de reciprocidad y motivarlos en favor del ejercicio de la ciudadanía y de la participación. ${ }^{8}$

8 Ciertamente, la intervención en ese nivel de conflictos y alianzas representa un desafío para el gestor, considerando el hecho de que tales desafíos no pueden ser sólo resueltos en el plano de la discursividad, 
Hoy, en el contexto de la actual sociedad de masa mundializada, la adopción de una comprensión más compleja de la familia es fundamental. Esta complejidad avanza en la reconfiguración de la familia como estructura simbólica y material, sin obedecer a una regla matemática rígida según sugería el estructuralismo lévi-straussiano (Lévi-Strauss 2003) que hizo negligente la plasticidad del comportamiento social presente en la tradición de Mauss, según recuerda Claude Lefort (1979). En ese sentido, gana importancia la idea de familia relacional, que extrapola la visión domiciliar al incorporar la red de parentesco (que refuerza el sentimiento grupal) y la red de parentela (que refuerza el sentimiento individual). La idea de familiared social crece en importancia "entre las personas más frágiles, para las cuales no se da un lugar en la sociedad y que no consiguen encontrarlo por sí mismas. Es la familia la que puede transmitirles un patrimonio de defensas internas" (Carvalho 2003:118). Es en ese escenario en el que las redes de solidaridad y de sociabilidad, engendradas a partir de la familia, ganan relevancia en la política social y sobre todo en la protección social movilizada en este final de siglo (Carvalho 2003:16). Planteamos, finalmente, que la familia-red en Brasil es una nueva actividad subpolítica desarrollada por la sociedad civil, actividad que Thumala y Cathalifaud (2006) estudian en la transformación social en Chile.

\section{Para concluir: aún la familia-red}

Algunos estudiosos del tema familia y red social (Portugal 1995) llaman la atención sobre el hecho de que la familia moderna, por el contrario de lo que se considera en la sociología clásica personificado en la figura de Parson, no está restringida a la 'familia nuclear', o sea, aquella de padres e hijos que viven bajo un mismo techo. Los estudiosos postulan que la unidad familiar característica de la sociedad industrial no es la 'familia nuclear aislada', sino la 'familia extensa modificada'. Esta difiere de la 'familia extensa clásica' en la medida en que no requiere de la proximidad geográfica o del nepotismo ocupacional, y está basada en relaciones igualitarias y no autoritarias; difiere de la 'familia nuclear aislada' en la medida en que existe un apoyo significativo al núcleo por parte de la red de parientes, distante social y fisicamente.

exigiendo un adentramiento en el plano de lo simbólico (donde están la identificación y el reconocimiento). No cabe profundizar sobre esta cuestión en el presente texto, pero vale la pena recordar que las experiencias originales e interesantes de intervención en el grupo familiar están siendo realizadas por profesionales de la psico-sociología, que trabajan a partir de un abordaje sistémico como es el caso, por ejemplo, de la teoría de la 'constelación familiar' sistematizada por el alemán Bert Hellinger, cuyo libro clásico sobre el asunto, ya traducido al portugués, se titula A simetria oculta do amor (1999). 
De esa forma, en caso de que se desee incitar a la participación social en las políticas públicas, primero que nada — nos recuerda Silvia Portugal (1995)— se debe tomar en consideración la red familiar y su inserción social, considerándose los tipos de lazos intrafamiliares y extrafamiliares (entre vecinos, amigos, asociados y miembros de la comunidad de pertenencia).

Por otra parte, se debe subrayar que la red social no es un fenómeno que se circunscriba a la familia, aunque tenga a esta última como referencia central para pensar la vida comunitaria y asociativa, y promueva el surgimiento del sujeto colectivo en la formación de la esfera pública. Con esto queremos afirmar que se torna relevante una concepción de familia de carácter sistémico y complejo, abierta a la integración con otras redes (vecindad, amistad y asociaciones), y capaz de superar los límites de una visión funcional que torna incomprensible el papel de las redes sociales primarias en la estructuración de las sociabilidades de los individuos en la esfera local, comunitaria y asociativa. En este sentido, el concepto de colaboración propuesto por Thumala y Arnold-Cathalifaud (2006), que destaca nuevas actitudes comunitarias de agentes que asumen su diversidad, individualidad y contingencia, nos parece adecuado para definir esos nuevos acuerdos de familia que mezclan la individualidad y la asociación.

Considerar a la familia en tanto red social o en cuanto fundamento de la vida asociativa, en el esfuerzo de avanzar en la descentralización e involucramiento de los actores locales en los programas públicos, superando la categoría restrictiva de la familia domicilio, implica tomar en cuenta la interferencia de los acuerdos familiares dominantes (familia únicamente constituida por miembros consanguíneos; familia constituida por miembros consanguíneos, pero parientes distantes y ahijados, etc.) y de los sistemas de reciprocidad dominantes (tipos de conflictos, alianzas y proyectos comunes) sobre las formas de las comunidades locales. Igualmente se precisa considerar la forma como esas últimas se organizan y reaccionan a los impactos de la acción pública. Esto es, la idea de familia-red no sustituye aquella de familia-domicilio: implica integrar una comprensión horizontal del modo de vida que extrapole el domicilio, y tal vez hasta la calle y el barrio, para focalizar las pulsiones del sujeto social.

Tal consideración significa que la acción pública debe tomar en cuenta la idea de familia en esta perspectiva socio-antropológica más amplia, que integra el domicilio pero lo supera al extenderse a las redes de trueques y reciprocidades entre los actores sociales locales. Esta es, a nuestro entender, la única manera de permitir el surgimiento de lo que Ruthy Laniado (2001) designa 'reciprocidad generalizada moderna'. Para esta autora, sólo los caracteres generalizador y altruista permiten crear vínculos más sólidos y reflexivos, y generar obligaciones solidarias más horizontales, siendo gran parte de esa solidaridad remitida a la esfera pública. 
Se puede observar que los valores y las normas sociales de la generosidad y de la reciprocidad son fundamentales para que el receptor de la acción pública se sienta deudor activo del juego democrático, dando seguimiento a otra acción de solidaridad en el plano horizontal. Vale decir, parte de la reciprocidad generalizada pasa a configurar, en el mundo socio-político, la obligación del ciudadano: derechos y deberes organizados en la esfera pública que disciplinan la relación entre público y privado, contribuyendo al surgimiento de formas públicas embrionarias (Caillé 2004a).

Esta es una posibilidad emancipadora provisoria para la implementación de la formulación del proyecto de revisión conceptual impostergable. Aquí, la voluntad del gestor público es decisiva, así como la de los liderazgos comunitarios y de los intelectuales.

\section{Referencias bibliográficas}

Angelo, M. y R.S. Bousso, 2001. "Fundamentos da assistência à família em saúde." Manual de enfermagem. Temas de caráter introdutório. Disponible en: www.ids-saude.org. br/enfermagem [febrero 2006].

Augusto, L.G., 2004. "Saúde, ambiente e desenvolvimento: desafios para a saúde pública." En: P.H. Martins y B. Fontes, eds. Redes sociais e saúde: novas possibilidades teóricas. Recife: Editora da Universidade Federal de Pernambuco, 93-102.

Bozon, M., 2004. Sociologia da sexualidade. Rio de Janeiro: Editora da Fundação Getúlio Vargas.

Caillé, A., 2004a. "Présentation." Revue du MAUSS semestrielle: De la reconnaissance. Don, identité et estime de soi, No 23, 5-28.

, 2004b. "A sociedade mundial no horizonte." En: P.H. Martins y B. Nunes, comps. A nova ordem social: perspectivas da solidariedade contemporânea. Brasília: Paralelo 15 Editores, 17-41.

Capra, F., 1998. A teia da vida: uma nova compreensão cientifica dos sistemas vivos. São Paulo: Cultrix.

Carvalho, I.M. y P.H. Almeida, 2003. "Família e proteção social.” São Paulo em perspectiva 17(2): 109-122.

Carvalho, M.C.B., 2003. “O lugar da família na política social.” En: M.C.B. Carvalho, ed. A família contemporânea em debate. São Paulo: Cortez / EDUC, 5-10.

Cavalcanti, H. y J. Burity, eds., 2002. Polifonia da miséria: uma construção de novos olhares. Recife: Massangana.

Chanial, P., 2004. "Todos direitos por todos e para todos: cidadania, solidariedade social e sociedade civil num mundo globalizado.” En: P.H. Martins y B. Nunes, eds. A nova 
ordem social: perspectivas da solidariedade contemporânea. Brasília: Paralelo 15 Editores, 58-70.

Dewey, J., 1997. The Public and its Problem. Athens: Ohio University Press.

Dowbor, L., 2003. A reprodução sócial: descentralização e participação, as novas tendências. Vol. III. Petrópolis: Vozes.

Durkheim, E., 1888. "Introduction à la sociologie de la famille." En: Annalles de la Faculté de Lettres de Bourdeaux. Paris: Les Éditions de Minuit, Collection Le Sens Commun 10, 257-281.

Fontes, B., 2004. "Capital social e terceiro setor: sobre a estruturação das redes sociais em associaçōes voluntárias.” En: P.H. Martins y B. Fontes, comps. Redes sociais e saúde: novas possibilidades teóricas. Recife: Editora da Universidade Federal de Pernambuco, 49-76.

Giddens, A., 1993. A transformação da intimidade: sexualidade, amor e erotismo nas sociedades modernas. São Paulo: Editora da Universidade Estadual de São Paulo.

Godbout, J., 2001. Le don, la dette et l'identité. Paris: La Découverte y MAUSS.

Graham, R., 1997. Clientelismo e política no Brasil no século XIX. Rio de Janeiro: Editora da Universidade Federal do Rio de Janeiro.

Godelier, M., 2004. Métamorphoses de la parenté. Paris: Fayard.

Heilborn, M.L., 2004a. Família e sexualidade. Rio de Janeiro: Editora da Fundação Getúlio Vargas.

2004b. Dois é par. Gênero e identidade sexual em contexto igualitário. Rio de Janeiro: Garamond Universitária.

Hellinger, B., 1999. A simetria oculta do amor. São Paulo: Cultrix.

James, W., 1981. Pragmatisme. Indianopolis, Cambridge: Hachett Publishing Company.

Jelin, E., 1994. "As famílias em América Latina.” En: Famílias: Siglo XXI. Santiago: ISIS Internacional, 157-198.

Laniado, R., 2001. "Troca e reciprocidade no campo da cultura política.” Sociedade e Estado: revista semestral de Sociologia, Vol. XVI, No 1-2, 222-244.

Lasch, C., 1990. O mínimo eu: sobrevivência psíquica em tempos difíceis. São Paulo: Brasiliense.

1991. Refúgio num mundo sem coração. A família: santuário ou instituição sitiada? Rio de Janeiro: Paz e Terra.

Leal, V. Nunes, 1986. Coronelismo, enxada e voto. São Paulo: Alfa-Ômega.

Lefort, C., 1979. "A troca e a luta dos homens.” En: C. Lefort, ed. As formas da história. São Paulo: Brasiliense.

Lévi-Strauss, C., 2003. As estruturas elementares do parentesco. Petrópolis: Vozes.

Martins, P.H., 2002. "Cultura autoritária e aventura da brasilidade.” En: J. Burity, comp. Cultura e identidade: perspectivas interdisciplinares. Rio de Janeiro: DP\&A, 65-100.

2004. "O Estado e a redistribuição dos bens da cidadania no contexto pósnacional." En: P.H. Martins y B. Nunes, comps. A nova ordem social: perspectivas da solidariedade contemporânea. Brasília: Paralelo 15 Editores. 
,2003. Contra a desumanização da medicina: crítica sociológica das práticas médicas modernas. Petrópolis: Vozes.

, 2004a. "As redes sociais, a dádiva e o paradoxo sociológico." En: P.H. Martins y B. Fontes, comps. Redes sociais e saúde: novas possibilidades teóricas. Recife: Editora da Universidade Federal de Pernambuco, 21-48.

2004c. "Pouvoir politique, action publique locale et économie solidaire." En: Magnen Laville França Filho y Medeiros, eds. Action publique et économie solidaire: une perspective internationale. Paris: Edition Eres.

2004d. "Gestão pública, reciprocidade e obrigação redistributivista: uma agenda para a reforma moral do Estado." Revista do Núcleo de Pós-Graduação e Pesquisa em Ciências Sociais da Universidade Federal de Sergipe, Año VII, No 7, 49-74.

2004e. "État, don et revenu de citoyenneté." Revue du MAUSS semestrielle: De la reconnaissance. Don, identité et estime de soi, No 23, 381-396.

Martins, P.H. y B. Fontes, 2004b. "Construindo o conceito de rede de vigilância em saúde.” En: P.H. Martins y B. Fontes, comps. Redes sociais e saúde: novas possibilidades teóricas. Recife: Editora da Universidade Federal de Pernambuco, 103-120.

Mauss, M., 2003a. "Uma categoria do espírito humano: a noção de pessoa, a de eu." En: M. Mauss, ed. Sociologia e Antropologia. São Paulo: Cosac \& Naify, 360-397. , 2003b. "Ensaio sobre a dádiva: forma e razão de troca nas sociedades arcaicas." En: M. Mauss, ed. Sociologia e Antropologia. São Paulo: Cosac \& Naify, 183-294.

Mello, S.L., 2003. "Família: perspectiva teórica e observação factual.” En: M.C. Sousa, comp. A familia contemporânea em debate. São Paulo: Cortez Editora/EDUC.

Mercklé, P., 2004. Sociologie des reseaux sociaux. Paris: La Découverte.

Moreno, J., 1987. Psychotérapie de groupe et psychodrame. Paris: Press Universitaire de France.

Morrison, A. y M.L. Biehl, 2000. A família ameaçada: violência doméstica nas Américas. Rio de Janeiro: Banco Interamericano de Desenvolvimento/Fundação Getúlio Vargas.

Porchmann, M., R. Amorim, 2003. Atlas da exclusão social no Brasil. São Paulo: Cortez.

Portugal, S., 1995. "As mãos que balancam o berço. Um estudo sobre redes informais de apoio a maternidade." Mimeo. Coimbra.

Queiroz, M.I., 1976. O mandonismo na vida política brasileira. São Paulo: Alfa-Ômega.

Ribeiro, E., 2004. "As várias abordagens da família no cenário do programa/estratégia de saúde da família (PSF). Revista Latino-Americana de Enfermagem, Vol. 12, No 4, 37-46.

Rodge, J., 1996. Family Life \& Social Control. A Sociological Perspective. London: MacMillan Press.

Rosanvallon, P., 1995. La crise de l'État-providence. Paris: Seuil. 1995. La nouvelle question sociale: repenser l'Etat-providence. Paris: Seuil.

Santos, B.S. y L. Avritzer, 2002. "Para ampliar o cânone democrático.” En: B.S. Santos, comp. Democratizar a democracia: os caminhos da democracia participativa. Rio de Janeiro: Civilização Brasileira. 
Sarti, C., 2003 "Família e individualidade: um problema moderno." En: M.C. Sousa, comp. A família contemporânea em debate. São Paulo: Cortez Editora/EDUC.

Schwartzman, S., 2004. As causas da pobreza. Rio de Janeiro: Editora da Fundação Getúlio Vargas.

Sennett, R., 2004. Respeito: a formação do caráter em um mundo desigual. São Paulo: Record.

Scott, R.P., 2005a. "Mulheres Chefes de Família: abordagens e temas para as políticas públicas." Mimeo. Recife.

2005b. "Família, gênero e poder no Brasil do século XX." Revista Anthropologica, Año 9, Vol. 16, 217-239.

Simmel, G., 1939. Sociología: estudios sobre las formas de socialización. Buenos Aires: Espasa Calpe.

Thumala, D. y M. Arnold-Cathalifaud, 2006. "Colaboración, participación y sociedad civil en Chile: proposiciones para la acción.” Resultados de la investigación "Colaboración, cultura y desarrollo", Dirección de Investigación de la Universidad de Chile (Proyecto DI SOC 04/14-2), fundamento al Observatorio de la Colaboración Social.

Wasserman, S. y K. Faust, 1994. Social Network Analysis. Methods and Applications. Cambridge: Cambridge University Press.

Williams, R., 1981. Culture. London: Fontana Press. 